\title{
Visit-to-visit variability of glycated albumin was associated with incidence or progression of lower extremity atherosclerotic disease
}

\author{
Yun Shen ${ }^{1,2+}$, Dongjun Dai ${ }^{1 \dagger}$, Jingyi Lu' ${ }^{1}$, Yufei Wang ${ }^{1}$, Wei Zhu' ${ }^{1}$, Yuqian Bao ${ }^{1}$, Gang Hu ${ }^{2^{*}}$ and Jian Zhou ${ }^{1 *}$ (D)
}

\begin{abstract}
Background: The aim of this study was to investigate the association of visit-to-visit variability of hemoglobin A1C (HbA1c) and glycated albumin (GA) with the risk of lower extremity atherosclerotic disease (LEAD).

Method: We performed a prospective cohort study of 436 patients with type 2 diabetes (258 men and 178 women) with at least 3 measurements of HbA1c and GA prior to baseline investigation from the Department of Endocrinology and Metabolism, Shanghai Sixth People's Hospital. Different HbA1c and GA variability markers were calculated. Multivariable Cox proportional hazard regression models were used to demonstrate the association between visit-to-visit $\mathrm{HbA} 1 \mathrm{c}$ and GA variability and the risk of incident or progressive LEAD.
\end{abstract}

Results: During a mean follow-up period of 3.77 years, 112 participants developed LEAD. Multivariate-adjusted hazard ratios (HRs) of LEAD across tertiles of GA-CV values were 1.00, 1.06 (95\% confidence interval [CI] 0.65-1.75), and 1.71 (95\% Cl 1.07-2.73) ( $P$ for trend $=0.042$ ), respectively. When we used GA-VIM and GA-ARV values as exposures, similar positive associations with the risk of LEAD primary were found. Multivariate-adjusted HRs of LEAD for each 1 unit increase in GA-CV, GA-VIM and GA-ARV were 1.03 (95\% Cl 1.01-1.06), 1.32 (95\% Cl 1.03-1.69), and 1.07 (95\%Cl 1.01-1.15), respectively. However, there was no significant association between visit-to-visit variability of HbA1c and the risk of LEAD.

Conclusions: Visit-to-visit variability of GA may be an optimal biomarker in relation to LEAD risk among patients with type 2 diabetes.

Keywords: Glycated albumin, Glycemic variability, Lower extremity atherosclerotic disease

\section{Background}

Lower extremity atherosclerotic disease (LEAD) is one of the life-threatening complications of diabetes. LEAD and the subsequent vascular occlusion, gangrene and lower extremity amputation seriously affect the quality of life of

\footnotetext{
*Correspondence: gang.hu@pbrc.edu; zhoujian@sjtu.edu.cn ${ }^{\dagger}$ Yun Shen and Dongjun Dai contributed equally to this work 'Department of Endocrinology and Metabolism, Shanghai Jiao Tong University Affiliated Sixth People's Hospital, Shanghai Clinical Center for Diabetes, Shanghai Diabetes Institute, Shanghai Key Laboratory of Diabetes Mellitus, 600 Yishan Road, Shanghai 200233, China ${ }^{2}$ Chronic Disease Epidemiology Laboratory, Pennington Biomedical Research Center, 6400 Perkins Road, Baton Rouge, LA 70808, USA
}

patients and increase the economic burden [1, 2]. However, the onset of LEAD is insidious, and $40 \%$ of patients are asymptomatic. Therefore, early identification and intervention to delay the progression of LEAD can effectively reduce the risk of the above serious outcomes.

Among patients with diabetes, long-term exposure to hyperglycemia is an important risk factor for the development of macrovascular complications. Glycated hemoglobin (HbA1c) has been widely used as a marker for glycemic control [3, 4]. Previously, we have demonstrated the association between visit-to-visit HbA1c variability and the incidence of both coronary heart disease and stroke by real world data. Severe hypoglycemia events

c) The Author(s) 2020. This article is licensed under a Creative Commons Attribution 4.0 International License, which permits use, sharing, adaptation, distribution and reproduction in any medium or format, as long as you give appropriate credit to the original author(s) and the source, provide a link to the Creative Commons licence, and indicate if changes were made. The images or other third party material in this article are included in the article's Creative Commons licence, unless indicated otherwise in a credit line to the material. If material is not included in the article's Creative Commons licence and your intended use is not permitted by statutory regulation or exceeds the permitted use, you will need to obtain permission directly from the copyright holder. To view a copy of this licence, visit http://creativeco mmons.org/licenses/by/4.0/. The Creative Commons Public Domain Dedication waiver (http://creativecommons.org/publicdomain/ zero/1.0/) applies to the data made available in this article, unless otherwise stated in a credit line to the data. 
may partly mediate the above association [5]. However, based on recent findings, HbA1c is insufficient in terms of the overall evaluation of glycemic control [6]. Recently, glycated albumin (GA) is also considered as an indicator that reflects long-term glycemic control. Compared with HbA1c, GA is not affected by the measurement of hemoglobin, and studies have found that GA may more accurately reflect long-term glycemic fluctuations in postprandial glucose levels than HbA1c [7], while most patients with diabetes in China are featured with postprandial hyperglycemia. In addition, cohort studies with long term follow up regarding LEAD as the primary outcome are limited.

Given the above reason, we speculate that visit-tovisit variability of GA can provide more information on long-term glycemic control than HbA1c. The association between visit-to-visit variability of GA and LEAD in patients with type 2 diabetes remains uncertain. The present study aimed to investigate the association between visit-to-visit variability of GA and the risk of LEAD in patients with type 2 diabetes.

\section{Methods \\ Study design, sample size calculation and participants}

This is a prospective and dynamic cohort study of patients with type 2 diabetes aged 18-80 years who were admitted to the Department of Endocrinology and Metabolism, Shanghai Sixth People's Hospital from January 2011 to September 2019. All patients were previously confirmed with type 2 diabetes by the 1999 World Health Organization criteria [8]. Eligible patients should have at least 3 measurements of GA and $\mathrm{HbA1c}$ within 2 years before baseline data collection. We calculated the sample size prior to the recruitment at baseline based on the findings from a pilot study. A Cox regression of the log hazard ratio on a covariate with a standard deviation of 7.3 of GA-CV based on a sample of 400 observations achieves $80 \%$ power at a 0.05 significance level to detect a regression coefficient equal to 0.04 . The sample size was adjusted for an anticipated event rate of 0.26 . Considering a $20 \%$ of loss during follow-ups, a total of 611 eligible patients with type 2 diabetes were screened at baseline to ensure a sufficient sample size. The average duration of diabetes was $11.5 \pm 6.49$ years. We excluded patients with a history of malignancy or mental disorders $(n=53)$, end-stage renal disease $(n=1)$, onset of diabetic ketoacidosis $(n=6)$, a history of congestive heart failure and lower extremity amputation at baseline $(\mathrm{n}=12)$. Finally, a total of 539 patients were enrolled in the study at baseline (see Additional file 1: Figure S1). The study and the analysis plan were approved by the Institutional Review Boards (Research Ethics Committees) of Shanghai Sixth
People's Hospital. We have obtained informed consent from all participants.

\section{Baseline anthropometric and laboratory measurements}

All participants underwent routine physical examination to measure height, body weight, waist circumference and blood pressure. Body mass index (BMI) was calculated as body weight in kilograms divided by square of height in meters. Waist circumference was measured by a tape around the horizontal plane between the inferior costal margin and the iliac crest on the mid-axillary line with the subject in a standing position. Blood pressure was measured twice with a mercury sphygmomanometer, and the average value of the two measurements was taken. History of comorbidities and prescriptions was collected including anti-hypertensive medications, glucose-lowering medications, lipid-lowering medications and antiplatelet medications. Using data collected on the patient's self-reported smoking status, we classified the patients into 2 groups: current smokers, non-current smokers including ever smokers and never smokers. Current alcohol consumption was defined as weekly alcohol consumption over $140 \mathrm{~g}$ in men and $70 \mathrm{~g}$ in women [9]. Mean value of HbA1c was defined as the one before the baseline data collection.

All subjects were fasted overnight for $8-10 \mathrm{~h}$ before venous blood sampling. Serum uric acid (UA), serum creatinine $(\mathrm{Scr})$, total cholesterol (TC), triglycerides (TG), low-density lipoprotein cholesterol (LDL-C), high density lipoprotein cholesterol (HDL-C), fasting blood glucose (FPG) and fasting C peptide (FCP) were measured using the same methods that have been previously described [10]. GA was measured via an enzymic method (Lucica GA-L, Asahi Kasei Pharma, Tokyo, Japan) with the 7600-120 auto-analyzer (Hitachi, Tokyo, Japan). The inter- and intra- batch coefficients of variation were $5.1 \%$ and $3.0 \%$ respectively. HbA1c was measured via high performance liquid chromatography (Variant II, Bio-Rad, Hercules, CA, USA). The inter- and intra- batch coefficients of variation were $3.4 \%$ and $2.6 \%$ respectively. The estimated glomerular filtration rate (eGFR) was estimated using the Chronic Kidney Disease Epidemiology Collaboration equation (CKD-EPI) [11].

\section{Visit-to-visit variability of GA and HbA1c}

The updated mean value, standard deviation (SD), variability independent of the mean (VIM), and the average real variability (ARV) of GA and HbA1c were calculated for each participant within 2 years before baseline date collection. $\mathrm{CV}$ of GA and HbA1c was calculated as $\mathrm{SD} /$ mean $\times 100 \%$. VIM was calculated as $\mathrm{SD} /$ mean $^{\beta}$. $\beta$ stands for a standardized coefficient of fitted power function with SD and mean. ARV was calculated as the 
average value of the difference between consecutive measurements [12]. In this study, the average numbers of measurements of GA and HbA1c during an average of 1.4 years before baseline were 3.6 and 3.8 , respectively.

\section{Prospective follow-up}

The primary outcome (LEAD) was the composite outcome of either incident LEAD or progressive LEAD confirmed by Doppler ultrasonography. The femoral artery, deep femoral artery, popliteal artery, superficial femoral artery, anterior tibial artery, posterior tibial artery and peroneal artery were measured bilaterally. Arterial intima-media thickness (IMT) was measured as the distance between the intima-lumen and the media-adventitia [13]. Atherosclerotic plaque was defined as a focal lesion that invaded the arterial lumen at least $0.5 \mathrm{~mm}$, or exceeded the surrounding IMT value by $50 \%$, or IMT $\geq 1.5 \mathrm{~mm}$ [14]. Patients with LEAD were confirmed by atherosclerotic plaque in any of the above-mentioned lower limb arterial segments [15]. Based on the above methodology, incident LEAD was defined as the development of new-onset atherosclerotic plaque in patients without LEAD at baseline. Progressive LEAD was defined as the expansion of lower extremity atherosclerotic plaque or the development of new lower extremity atherosclerotic occlusion in patients with LEAD at baseline. The secondary outcomes included incident LEAD and progressive LEAD individually. The duration of follow-up for each patient was calculated from the baseline date to the date of diagnosis of the outcome, the date of dropout, the date of death, or June 30, 2020.

\section{Statistical analyses}

The associations between visit-to-visit variability of GA and HbA1c (CV, VIM and ARV) and the risk of the primary outcome were analyzed by use of Cox proportional hazards models. CV, VIM and ARV of GA and CV of HbA1c were evaluated in the following 2 ways: as tertiles and as a continuous variable (per 1-unit increase). These visit-to-visit GA and HbA1c variability metrics were included in the models as dummy variables, and the significance of the trend across categories of GA and HbAlc was tested in the same models by giving an ordinal numeric value for each dummy variable. The proportional hazards assumption in the Cox model was assessed with graphical methods and with models including time-by-covariate interactions. In general, all proportionality assumptions were appropriate. All analyses were conducted after adjusting for age and sex, and then a backward stepwise selection procedure was used for candidate covariates in the multivariable model including BMI, waist circumference, diabetes duration, systolic blood pressure (SBP), lipid profile (TG, LDL-C and
HDL-C), eGFR, smoking status, current alcohol drinking, insulin therapy, anti-hypertensive therapy, aspirin, statins therapy, and mean value of HbA1c. Finally, we included diabetes duration, smoking status, eGFR, HDL-C, mean value of $\mathrm{HbA} 1 \mathrm{c}$ and use of aspirin in the multivariableadjusted model. Statistical significance was considered to be $P<0.05$. All statistical analyses were performed using IBM SPSS Statistics for Windows, version 24.0 (IBM Corp., Armonk, NY, USA).

\section{Results}

A total of 539 patients were enrolled in the study at baseline with a loss of 103 during follow-ups. Finally, 436 patients with type 2 diabetes were included into the final analysis. Compared with patients with diabetes excluded in the present study, the patients with type 2 diabetes included in the present study had the similar age (58.6 versus 59.9 years old, $P=0.324)$, and fewer men $(59.2 \%$ versus $67.0 \%, P<0.05)$. The baseline characteristics are listed in Table 1 based on the presence of LEAD at baseline. Patients with LEAD at baseline were older, with longer duration of diabetes, and had higher systolic BP, worse renal function, lower total cholesterol, and were more likely to receive insulin, aspirin and statin therapies compared with patients without LEAD at baseline.

During a mean follow-up period of 3.77 years, 112 participants developed the primary outcome. Multivariateadjusted (diabetes duration, smoking status, eGFR, HDL, aspirin, and mean value of HbA1c) HRs of the primary outcome across tertiles of GA-CV values were 1.00, 1.06 (95\% confidence interval [CI] 0.65-1.75), and 1.71 (95\% CI 1.07-2.73) ( $P$ for trend $=0.042$ ), respectively (Table 2). When we used GA-VIM and GA-ARV values as exposures, similar positive associations with the risk of LEAD were found (Table 2). Multivariate-adjusted HRs of LEAD for each 1 unit increase in GA-CV, GA-VIM and GA-ARV were 1.03 (95\% CI 1.01-1.06), 1.32 (95\% CI 1.03-1.69), and 1.07 (95\%CI 1.01-1.15), respectively (Table 2). Figure 1a presented the significant trend of hazard ratios for the primary outcome across tertiles of GA-CV.

Hazard ratios of the primary outcome at different time points are listed in Table 3. The multivariable-adjusted hazard ratios of LEAD by GA-CV within different follow-up years were 0.99 (95\% CI 0.91-1.07) within 1 year, 1.02 (95\% CI $0.98-1.06)$ within 2 years, 1.02 (95\%CI $0.99-1.05)$ within 3 years, 1.03 (95\% CI 1.01-1.06) within in 4 years, $1.03(95 \%$ CI $1.01-1.05)$ within 5 years, 1.03 (1.01-1.05) within 6 years, and 1.03 (95\% CI 1.01-1.06) for 6 years and over, respectively. However, both GAVIM and GA-ARV were not reflective of the primary outcome in assessing the risks of the primary outcome at different time points (Table 3 ). 
Table 1 Baseline characteristics of study participants

\begin{tabular}{|c|c|c|c|c|}
\hline Variables & All subjects & $\begin{array}{l}\text { With LEAD } \\
\text { at baseline }\end{array}$ & $\begin{array}{l}\text { Without LEAD } \\
\text { at baseline }\end{array}$ & $P$ value \\
\hline No. of subjects & 436 & 194 & 242 & - \\
\hline Male, $\%$ & 59.2 & 64.9 & 52.1 & 0.007 \\
\hline Age, years & $58.6 \pm 10.3$ & $63.4 \pm 7.92$ & $52.6 \pm 9.65$ & $<0.001$ \\
\hline Diabetes duration, years & $11.7 \pm 6.51$ & $13.4 \pm 6.55$ & $9.63 \pm 5.84$ & $<0.001$ \\
\hline Body mass index, $\mathrm{kg} / \mathrm{m}^{2}$ & $25.4 \pm 2.98$ & $25.3 \pm 3.08$ & $25.5 \pm 2.86$ & 0.583 \\
\hline Waist, $\mathrm{cm}$ & $92.0 \pm 8.92$ & $92.5 \pm 8.68$ & $91.3 \pm 9.20$ & 0.154 \\
\hline Systolic blood pressure, $\mathrm{mmHg}$ & $133 \pm 16.1$ & $135 \pm 16.7$ & $128 \pm 14.8$ & $<0.001$ \\
\hline Diastolic blood pressure, $\mathrm{mmHg}$ & $79.2 \pm 8.65$ & $79.2 \pm 8.51$ & $79.3 \pm 8.85$ & 0.867 \\
\hline $\mathrm{HbA} 1 \mathrm{c}, \mathrm{mmol} / \mathrm{mol}$ & $63.8 \pm 17.1$ & $64.7 \pm 16.2$ & $62.6 \pm 18.1$ & 0.188 \\
\hline $\mathrm{HbA} 1 \mathrm{c}, \%$ & $7.99 \pm 1.57$ & $8.08 \pm 1.49$ & $7.88 \pm 1.66$ & 0.188 \\
\hline $\mathrm{GA}, \%$ & $19.8 \pm 5.81$ & $20.0 \pm 5.58$ & $19.6 \pm 6.04$ & 0.566 \\
\hline Fasting Plasma glucose, $\mathrm{mmol} / \mathrm{L}$ & $7.62 \pm 2.41$ & $7.48 \pm 2.27$ & $7.80 \pm 2.56$ & 0.174 \\
\hline Fasting C-peptide, ng/mL & $2.10 \pm 1.53$ & $2.02 \pm 1.06$ & $2.19 \pm 1.96$ & 0.248 \\
\hline Current smoker, $\%$ & 27.5 & 29.3 & 25.3 & 0.343 \\
\hline Alcohol drinker, $\%$ & 13.1 & 14.0 & 11.9 & 0.499 \\
\hline $\mathrm{eGFR}, \mathrm{mL} /\left(\min 1.73 \mathrm{~m}^{2}\right)$ & $93.6 \pm 17.9$ & $87.7 \pm 17.6$ & $101 \pm 15.2$ & $<0.001$ \\
\hline Uric acid, $\mu \mathrm{mol} / \mathrm{L}$ & $335 \pm 83.1$ & $341 \pm 83.7$ & $330 \pm 80.8$ & 0.169 \\
\hline Total cholesterol, mmol/L & $4.48 \pm 1.15$ & $4.35 \pm 1.30$ & $4.64 \pm 0.90$ & 0.007 \\
\hline Triglyceride, mmol/L & $1.85 \pm 2.36$ & $1.79 \pm 2.82$ & $1.93 \pm 1.61$ & 0.534 \\
\hline LDL cholesterol, mmol/L & $2.61 \pm 0.88$ & $2.54 \pm 0.95$ & $2.70 \pm 0.79$ & 0.051 \\
\hline HDL cholesterol, mmol/L & $1.08 \pm 0.30$ & $1.06 \pm 0.27$ & $1.11 \pm 0.33$ & 0.093 \\
\hline \multicolumn{5}{|l|}{ Use antidiabetes agents, \% } \\
\hline Biguanides & 49.5 & 43.0 & 57.7 & 0.002 \\
\hline Sulfonylureas & 33.1 & 31.0 & 37.6 & 0.146 \\
\hline Thiazolidinediones & 8.94 & 7.85 & 10.3 & 0.371 \\
\hline Glinides & 13.1 & 10.3 & 16.5 & 0.058 \\
\hline DPP-4 inhibitors & 4.59 & 4.55 & 4.64 & 0.963 \\
\hline Glucosidase inhibitors & 62.8 & 68.6 & 55.7 & 0.006 \\
\hline SGLT-2 inhibitors & 0.00 & 0.00 & 0.00 & - \\
\hline GLP-1 receptor agonists & 1.15 & 0.41 & 2.06 & 0.108 \\
\hline Insulin, \% & 61.7 & 66.1 & 56.2 & 0.034 \\
\hline \multicolumn{5}{|l|}{ Use antihypertension agents, \% } \\
\hline RAAS inhibitors & 48.9 & 55.4 & 40.7 & 0.002 \\
\hline Calcium-channel blockers & 30.7 & 36.0 & 24.2 & 0.008 \\
\hline$\beta$-Blockers & 18.8 & 22.7 & 13.9 & 0.019 \\
\hline Diuretics & 6.65 & 10.7 & 1.55 & $<0.001$ \\
\hline \multicolumn{5}{|l|}{ Use lipid-lowering agents, \% } \\
\hline Statins & 56.2 & 66.9 & 42.8 & $<0.001$ \\
\hline Fibrates & 7.57 & 6.20 & 9.28 & 0.237 \\
\hline Aspirin, \% & 49.8 & 60.7 & 36.1 & $<0.001$ \\
\hline Anticoagulant, \% & 0.00 & 0.00 & 0.00 & - \\
\hline Mean $\mathrm{HbA} 1 \mathrm{c}, \mathrm{mmol} / \mathrm{mol}$ & $62.9 \pm 15.1$ & $64.4 \pm 14.9$ & $61.1 \pm 15.1$ & 0.025 \\
\hline Mean $\mathrm{HbA} 1 \mathrm{c}, \%$ & $7.91 \pm 1.38$ & $8.05 \pm 1.36$ & $7.75 \pm 1.38$ & 0.025 \\
\hline Mean GA, \% & $19.7 \pm 4.70$ & $20.0 \pm 4.66$ & $19.3 \pm 4.72$ & 0.101 \\
\hline \multicolumn{5}{|l|}{ Variability of GA } \\
\hline$C V$ & $11.1 \pm 7.20$ & $11.9 \pm 6.90$ & $10.2 \pm 7.48$ & 0.017 \\
\hline VIM & $1.03 \pm 0.74$ & $1.11 \pm 0.72$ & $0.94 \pm 0.75$ & 0.022 \\
\hline ARV & $3.09 \pm 2.45$ & $3.27 \pm 2.40$ & $2.86 \pm 2.48$ & 0.085 \\
\hline
\end{tabular}


Table 1 (continued)

\begin{tabular}{llll}
\hline Variables & All subjects & $\begin{array}{l}\text { With LEAD } \\
\text { at baseline }\end{array}$ & $\begin{array}{l}\text { Without LEAD } \\
\text { at baseline }\end{array}$ \\
\hline Variability of HbA1C & & & $P$ value \\
CV & $7.44 \pm 5.59$ & $7.53 \pm 5.54$ & $7.34 \pm 5.66$ \\
VIM & $0.23 \pm 0.18$ & $0.23 \pm 0.18$ & $0.22 \pm 0.18$ \\
ARV & $0.80 \pm 0.72$ & $0.81 \pm 0.69$ & $0.79 \pm 0.76$ \\
\hline
\end{tabular}

Table 2 Hazard ratios for the primary outcome based on different GA variability metrics

\begin{tabular}{|c|c|c|c|c|c|}
\hline & \multicolumn{3}{|c|}{ GA variability indicators tertiles } & \multirow[t]{2}{*}{$P$ value for trend } & \multirow[t]{2}{*}{ Per 1 unit increase } \\
\hline & T1 & $\mathrm{T} 2$ & T3 & & \\
\hline CV & $<7.35$ & $7.35-12.47$ & $\geq 12.48$ & - & - \\
\hline No. of participants & 144 & 145 & 147 & - & - \\
\hline No. of cases & 31 & 32 & 49 & - & - \\
\hline Person-years & 510 & 507 & 475 & - & - \\
\hline Age and sex-adjusted HRs & 1.00 & $1.02(0.62-1.68)$ & $1.74(1.10-2.73)$ & 0.019 & $1.03(1.01-1.06)$ \\
\hline Multivariable adjusted HRs & 1.00 & $1.06(0.65-1.75)$ & $1.71(1.07-2.73)$ & 0.042 & $1.03(1.01-1.06)$ \\
\hline VIM & $<0.61$ & $0.61-1.12$ & $\geq 1.13$ & - & - \\
\hline No. of participants & 146 & 144 & 146 & - & - \\
\hline No. of cases & 32 & 31 & 49 & - & - \\
\hline Person-years & 521 & 521 & 451 & - & - \\
\hline Age and sex-adjusted HRs & 1.00 & $0.95(0.58-1.57)$ & $1.85(1.19-2.89)$ & 0.004 & $1.40(1.12-1.76)$ \\
\hline Multivariable adjusted HRs & 1.00 & $0.99(0.60-1.64)$ & $1.65(1.02-2.67)$ & 0.050 & $1.32(1.03-1.69)$ \\
\hline ARV & $<1.75$ & $1.75-3.29$ & $\geq 3.30$ & - & - \\
\hline No. of participants & 145 & 146 & 145 & - & - \\
\hline No. of cases & 29 & 33 & 50 & - & - \\
\hline Person-years & 510 & 523 & 460 & - & - \\
\hline Age and sex-adjusted HRs & 1.00 & $1.11(0.67-1.82)$ & $1.97(1.24-3.12)$ & 0.005 & $1.10(1.03-1.18)$ \\
\hline Multivariable adjusted HRs & 1.00 & $1.08(0.64-1.81)$ & $1.81(1.08-3.04)$ & 0.032 & $1.07(1.01-1.15)$ \\
\hline
\end{tabular}

Multivariable adjustments included diabetes duration, smoking status, eGFR, HDL, aspirin, and mean $\mathrm{HbA} 1 \mathrm{c}$

When stratified analyses were utilized, the positive association between GA-CV and the risk of the primary outcome was consistent among most but not all of subgroups (Fig. 2). Significant associations between GA-CV and the primary outcome were detected among patients with current smoking status, current alcohol drinking status, antihypertensive medication, use of aspirin and without hyperuricemia.

Associations between $\mathrm{HbA1c}-\mathrm{CV}$ and the risks of the primary outcome are presented in Additional file 1: Table S1. There was no significant association between visit-to-visit variability of HbA1c and the risk of the primary outcome.

For the analysis of the secondary outcome (Additional file 1: Table S2), although the trends across tertiles of GA variability metrics in relation to incident LEAD were not significant (all $P>0.05$ ), the linear association between GA-CV, GA-VIM and incident LEAD remained significant with multivariable adjustments (HR 1.04 (1.01-1.07) for CV and 1.44 (1.05-1.97) for VIM). Similar results were observed for the outcome of progressive LEAD with additionally GA-ARV significantly associated with the outcome as well.

\section{Discussion}

In this prospective cohort study, we reported the positive association of long-term impact of GA-CV with the risk of LEAD among patients with type 2 diabetes, independent of HbA1c. We provided new insights into the clinical interpretation and evidence of GA. GA-CV may be a suitable metric for long-term glycemic variability, and it can be used as a supplementary measurement to conventional glycemic markers (such as mean glucose and $\mathrm{HbA1c}$ ) during the comprehensive management of diabetic complications. 

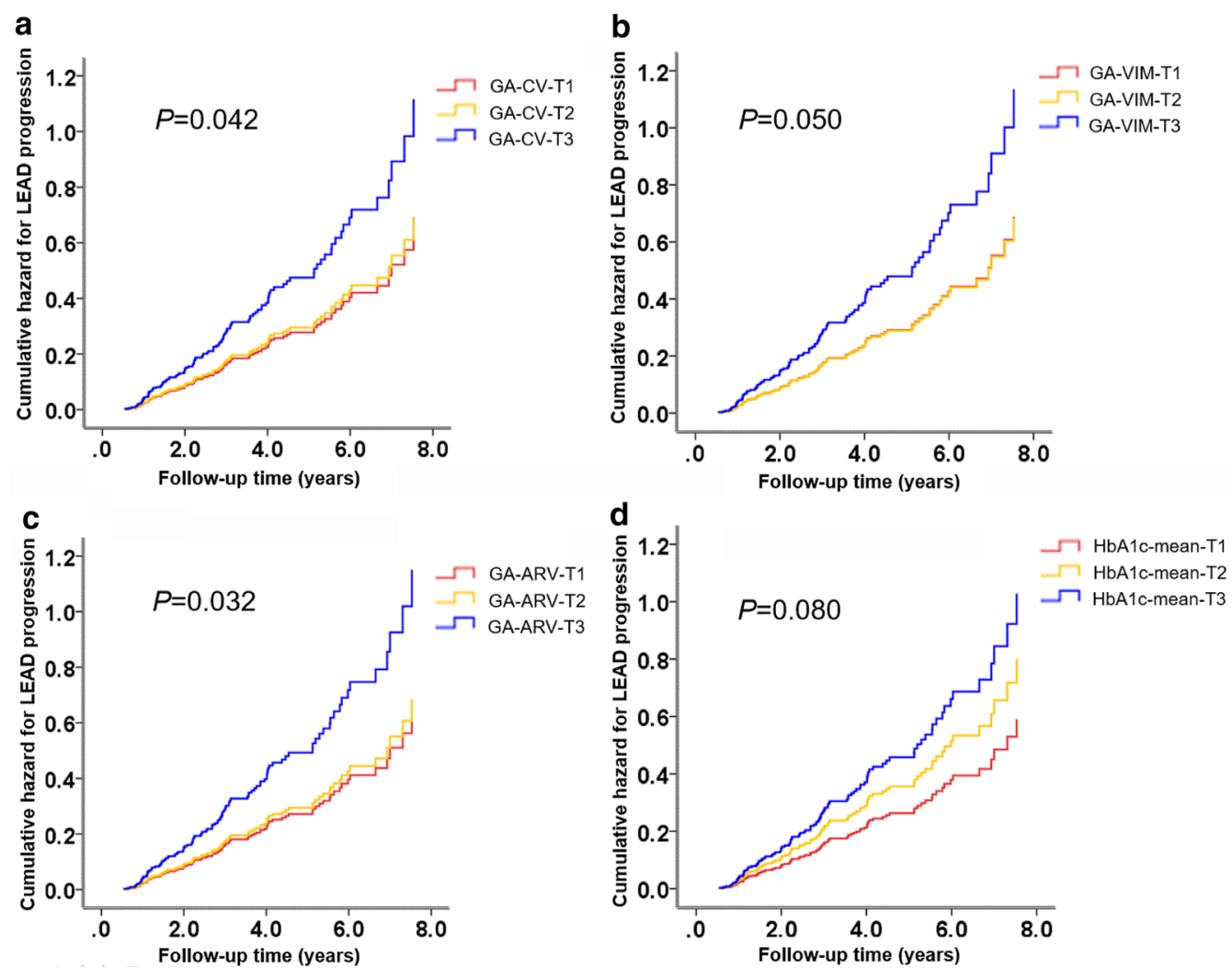

Fig. 1 Cumulative incidences of the LEAD progression according to the GA variability indicators (1A-CV, 1B-VIM, 1C-ARV) and mean A1C (1D) tertiles (T1-T3) using the cox proportional hazards regression analysis

\section{Clinical implications of GA and GA variability in other studies}

GA itself was reported to be closely related to cardiovascular disease and its subtypes in patients with diabetes [16] and even among subjects without diabetes or those with normal HbA1c levels [17], suggesting that GA has a potential advantage in predicting diabetic macrovascular complications. Moreover, GA may have other clinical implications other than diabetic complications such as a potential biomarker to predict the effects of clopidogrel antiplatelet therapy in ACS patients [18]. Limited evidence supported the association between visit-to-visit variability of GA and diabetic complications. June et al. [19] enrolled 498 patients with type 2 diabetes for more than 2 years of follow-up, and found that visit-to-visit variability of GA was significantly correlated with cardiac autonomic neuropathy. Another study involving 369 patients with type 2 diabetes by Park et al. [20] found that a higher visit-tovisit variability of GA was associated with a higher risk of diabetic nephropathy. Here, we demonstrated a significant association between GA variability and LEAD, which has broadened the clinical implications of GA.
Meanwhile, survival analysis at different time points showed that GA variability may have a potential impact on LEAD within 4 years and over, indicating that this is an optimal long-term biomarker.

\section{Advantages of $\mathrm{GA}$ when compared to $\mathrm{HbA1c}$}

Compared with HbA1c, GA is not affected by hemoglobin, and can more accurately reflect the glycemic control in cases of anemia, hemoglobinopathy, dialysis [21], and pregnancy [22]. GA can reflect glycemic control in a relatively short period of time (2-3 weeks) when blood glucose deteriorates and improves alternately within a short period of time. HbA1c often remains unchanged while GA is reflective of this kind of glycemic fluctuation, suggesting that multiple measurements of GA are comparable to HbA1c with more information about long-term glycemic fluctuations. Koga et al. [7] regularly measured the GA and HbA1c values of patients with diabetes within one year and calculated the CV of these two metrics respectively. They found that GA-CV was significantly higher than $\mathrm{HbA1c}-\mathrm{CV}$, which was similar to our findings. 
Table 3 Hazard ratios for the primary outcome at different time intervals

\begin{tabular}{|c|c|c|c|}
\hline & GA-CV & GA-VIM & GA-ARV \\
\hline No. of participants & 436 & 436 & 436 \\
\hline \multicolumn{4}{|l|}{$<1$ year } \\
\hline No. of cases & 13 & 13 & 13 \\
\hline Person-years & 298 & 298 & 298 \\
\hline Age-adjusted HRs & $0.97(0.90-1.05)$ & $0.85(0.40-1.78)$ & $1.01(0.83-1.24)$ \\
\hline Multivariable adjusted HRs & $0.99(0.91-1.07)$ & $0.97(0.44-2.12)$ & $1.05(0.85-1.30)$ \\
\hline \multicolumn{4}{|l|}{$<2$ years } \\
\hline No. of cases & 44 & 44 & 44 \\
\hline Person-years & 543 & 543 & 543 \\
\hline Age-adjusted HRs & $1.02(0.98-1.06)$ & $1.21(0.84-1.74)$ & $1.09(0.98-1.20)$ \\
\hline Multivariable adjusted HRs & $1.02(0.98-1.06)$ & $1.18(0.79-1.75)$ & $1.08(0.97-1.21)$ \\
\hline \multicolumn{4}{|l|}{$<3$ years } \\
\hline No. of cases & 73 & 73 & 73 \\
\hline Person-years & 725 & 725 & 725 \\
\hline Age-adjusted HRs & $1.03(0.99-1.06)$ & $1.33(1.01-1.77)$ & $1.10(1.01-1.19)$ \\
\hline Multivariable adjusted HRs & $1.02(0.99-1.05)$ & $1.22(0.90-1.66)$ & $1.06(0.97-1.16)$ \\
\hline \multicolumn{4}{|l|}{$<4$ years } \\
\hline No. of cases & 88 & 88 & 88 \\
\hline Person-years & 852 & 852 & 852 \\
\hline Age-adjusted HRs & $1.03(1.01-1.06)$ & $1.33(1.05-1.75)$ & $1.10(1.03-1.18)$ \\
\hline Multivariable adjusted HRs & $1.03(1.01-1.06)$ & $1.30(0.99-1.70)$ & $1.07(0.99-1.15)$ \\
\hline \multicolumn{4}{|l|}{$<5$ years } \\
\hline No. of cases & 95 & 95 & 95 \\
\hline Person-years & 929 & 929 & 929 \\
\hline Age-adjusted HRs & $1.03(1.01-1.05)$ & $1.36(1.07-1.73)$ & $1.10(1.03-1.18)$ \\
\hline Multivariable adjusted HRs & $1.03(1.01-1.05)$ & $1.27(0.98-1.64)$ & $1.07(0.99-1.15)$ \\
\hline \multicolumn{4}{|l|}{$<6$ years } \\
\hline No. of cases & 106 & 106 & 106 \\
\hline Person-years & 976 & 976 & 976 \\
\hline Age-adjusted HRs & $1.03(1.01-1.06)$ & $1.39(1.11-1.75)$ & $1.10(1.03-1.18)$ \\
\hline Multivariable adjusted HRs & $1.03(1.01-1.05)$ & $1.29(1.01-1.66)$ & $1.06(0.99-1.14)$ \\
\hline \multicolumn{4}{|l|}{$\geq 6$ years } \\
\hline No. of cases & 112 & 112 & 112 \\
\hline Person-years & 1006 & 1006 & 1006 \\
\hline Age-adjusted HRs & $1.03(1.01-1.06)$ & $1.40(1.12-1.76)$ & $1.10(1.03-1.18)$ \\
\hline Multivariable adjusted HRs & $1.03(1.01-1.06)$ & $1.32(1.03-1.69)$ & $1.07(1.01-1.15)$ \\
\hline
\end{tabular}

Multivariable adjustments included diabetes duration, smoking status, eGFR, HDL, aspirin, and mean $\mathrm{HbA1c}$

\section{Comparisons of different metrics of glycemic variability}

We calculated several metrics in the present study including CV, VIM and ARV of GA and HbA1c based on at least 3 measurements within 2 years prior to baseline as indicators for evaluating long-term glycemic fluctuations. Notably, SD, CV, adj-SD, VIM, ARV and variability scores have all been used in previous studies [12, 23, 24]. Of these metrics, SD and adjSD are affected by the average value and cannot fully reflect the glycemic variability; $\mathrm{CV}$ is not affected by the average value and is an ideal indicator for glycemic variability; VIM adjusts the effect of the average value on SD. There is a close association between ARV and variability scores, while variability score requires more times of measurement. Therefore, CV, VIM and ARV were selected as the exposures in the present study. Our findings supported GA-CV as a competitive marker in relation to LEAD, especially among type 2 diabetes patients with a moderate baseline level of HbA1c (mean level around 8\%). 


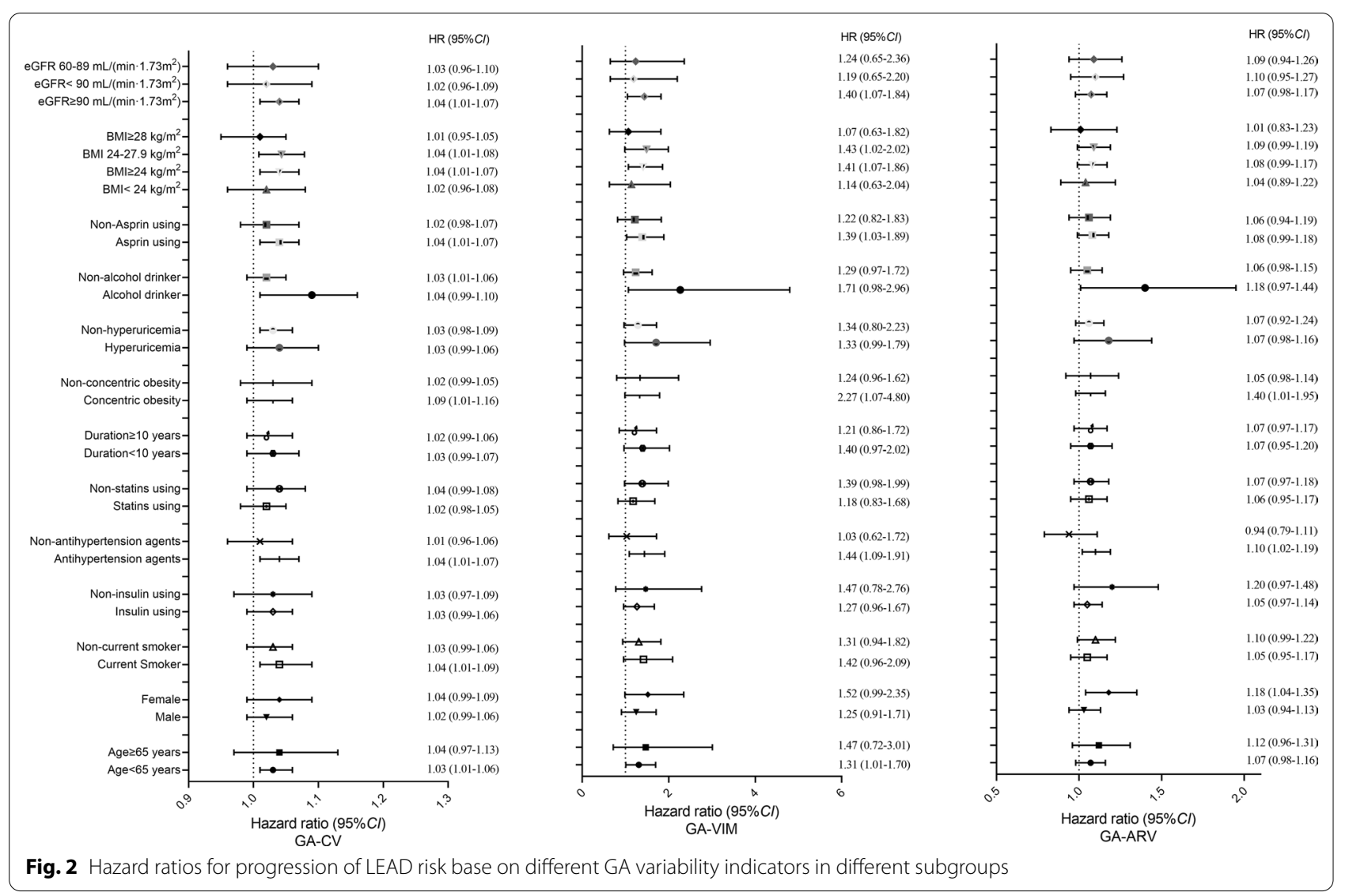

\section{Underlying mechanisms and potential bias of GA}

The mechanism of an increased risk of macrovascular complications caused by increased long-term blood glucose fluctuations is not yet clear. Studies have potentially supported the hypothesis of oxidative stress [25], endothelial dysfunction [26] and the subsequent chronic inflammation caused by blood glucose variability. Basic scientists have shown that exposure to oscillating glucose was more deleterious than constant high glucose and induced a metabolic memory after glucose normalization [27]. This may partly explain why a patient with larger glycemic variability may be more likely to develop diabetic complications than one with constant hyperglycemia even when they have a same value of HbA1c. Although socioeconomic factors were not assessed in this study, we may assume a large discrepancy in these factors such as adherence to treatment, self-efficacy in diabetes management, and quality of life between patients with and without a stable glycemic control [28]. However, two studies have raised concerns about the potential impact of visceral adiposity on GA. Measurements of GA may be underestimated among obese patients, especially among those with visceral obesity [29, 30]. To address this concern, we have considered waist circumference as a potential confounding factor in the analysis. Waist circumference was not included in the model when we used a backward stepwise selection procedure. It seemed that visceral adiposity was not involved in the association between GA and LEAD in this study, but we expect more evidence supporting our findings.

\section{Strengths and limitations}

The strength of this study included the originality, the prospective study design and the robust analysis. Limited studies have investigated the association between visit-to-visit variability of GA and the risk of LEAD in patients with type 2 diabetes. There are some limitations that need to be pointed out. First, this was a single center study with a relatively small sample size and few outcome cases. Second, our analyses adjusted for some confounding factors, but unmeasured factors such as other related chronic diseases, dietary factors, and physical activity could not be evaluated. Moreover, the index date of the primary outcome was determined by the date of lower extremity vascular ultrasonography. It is thus not yet possible to accurately obtain the time of lesion progression. However, the median follow-up time interval (Q1, Q3) in this study was $1.14(0.85,1.97)$ years, which is in line with 
the screening frequency recommended in guidelines for patients under a high risk of LEAD [31]. The subjects of this study were patients who had 3 or more HbA1c and GA measurements within 2 years, and had regular lower limb vascular ultrasonographic follow-ups. The compliance of this cluster of patients was higher than that of the general population with type 2 diabetes, which may account for a potential selection bias. Further retrospective real-world data with a large sample size are of great interest to validate our findings.

\section{Conclusions}

In conclusion, visit-to-visit variability of GA predicts LEAD risk among patient with type 2 diabetes, independent of HbA1c levels. In contrast, visit-to-visit variability of $\mathrm{HbA1c}$ was not an independent factor for LEAD among patients with type 2 diabetes. Visit-to-visit variability of GA may be closely related to LEAD among patients with type 2 diabetes.

\section{Supplementary Information}

The online version contains supplementary material available at https://doi. org/10.1186/s12933-020-01187-1.

Additional file 1: Table S1. Hazard ratios for the primary outcome based on different $\mathrm{HbA1}$ c variability metrics. Table $\mathbf{S 2}$. Hazard ratios for the secondary outcome based on different GA variability metrics. Figure S1. Flow chart of the study population.

\section{Abbreviations}

ARV: Average real variability; BMI: Body mass index; Cl: Confidence interval; CKD-EPI: Chronic Kidney Disease Epidemiology Collaboration equation; CV: Coefficient of variation; DPP-4: Dipeptidyl peptidase-4; eGFR: Estimated glomerular filtration rate; FCP: Fasting C peptide; FPG: Fasting blood glucose; GA: Glycated albumin; GLP-1: Glucagon-like peptide-1; HbA1c: Glycated hemoglobin A1c; HDL-C: High density lipoprotein cholesterol; HR: Hazard ratios; IMT: Intima-media thickness; LDL-C: Low-density lipoprotein cholesterol; LEAD: Lower extremity atherosclerotic disease; RAAS: Renin-AngiotensinAldosterone System; SBP: Systolic blood pressure; Scr: Serum creatinine; SD: Standard deviation; SGLT-2: Sodium-dependent glucose transporters-2; TC: Total cholesterol; TG: Triglycerides; UA: Uric acid; VIM: Variability independent of the mean.

\section{Acknowledgements}

We would like to thank all the involved clinicians, nurses, and technicians for helping with the study. We are grateful to all participants for their dedication in data collection and laboratory measurements.

\section{Authors' contributions \\ $J Z$ and GH conceived and designed the study. YS, DD and JL contributed to data collection, data analysis, and writing the paper. YS and DD contributed to data analysis. YW and WZ contributed to conduction of study and data collection. YB and JZ contributed to interpretation of data and revision of the manuscript. GH contributed to the revision of the manuscript. All authors revised the manuscript for important intellectual content and have approved the final version. All authors read and approved the final manuscript.}

\section{Funding}

This work was funded by the National Key R\&D Program of China (2018YFC2000802), the Shanghai Municipal Education Commission—Gaofeng Clinical Medicine Grant Support (20161430) and Shanghai Municipal Key Clinical Specialty.

\section{Availability of data and materials}

The datasets generated and/or analyzed in the current study are not publicly available but are available from the corresponding author on reasonable request.

\section{Ethics approval and consent to participate}

The study and the analysis plan were approved by the Institutional Review Boards (Research Ethics Committees) of Shanghai Sixth People's Hospital. We have obtained informed consent from all participants.

\section{Consent for publication}

All authors have read and approved submission of abstract and the abstract has not been published and is not being considered for publication elsewhere in whole or part in any language.

\section{Competing interests}

The authors declare that they have no competing interests.

Received: 14 October 2020 Accepted: 30 November 2020

Published online: 10 December 2020

\section{References}

1. Firnhaber JM, Powell CS. Lower extremity peripheral artery disease: diagnosis and treatment. Am Fam Physician. 2019;99(6):362-9.

2. Pourghaderi P, Yuquimpo KM, Roginski Guetter C, Mansfeld L, Park $\mathrm{HS}$. Outcomes following lower extremity amputation in patients with diabetes mellitus and peripheral arterial disease. Ann Vasc Surg. 2020;63:259-68.

3. Diabetes Control and Complications Trial Research Group, Nathan DM, Genuth S, Lachin J, Cleary P, Crofford O, et al. The effect of intensive treatment of diabetes on the development and progression of long-term complications in insulin-dependent diabetes mellitus. N Engl J Med. 1993:329(14):977-86.

4. UK Prospective Diabetes Study (UKPDS) Group. Intensive blood-glucose control with sulphonylureas or insulin compared with conventional treatment and risk of complications in patients with type 2 diabetes (UKPDS 33). UK Prospective Diabetes Study (UKPDS) Group. Lancet. 1998;352(9131):837-53.

5. Beyond A1C Writing Group. Need for regulatory change to incorporate beyond A1C glycemic metrics. Diabetes Care. 2018;41(6):e92-4.

6. Shen Y, Zhou J, Shi L, Nauman E, Katzmarzyk PT, Price-Haywood EG, et al. Association between visit-to-visit $\mathrm{HbA1c}$ variability and the risk of cardiovascular disease in patients with type 2 diabetes. Diabetes Obes Metab. 2020. Online ahead of print.

7. Koga M, Murai J, Morita S, Saito H, Kasayama S. Comparison of annual variability in $\mathrm{HbA} 1 \mathrm{C}$ and glycated albumin in patients with type $1 \mathrm{vs}$. type 2 diabetes mellitus. J Diabetes Complications. 2013;27(3):211-3.

8. Alberti KG, Zimmet PZ. Definition, diagnosis and classification of diabetes mellitus and its complications. Part 1: diagnosis and classification of diabetes mellitus provisional report of a WHO consultation. Diabet Med. 1998;15(7):539-53.

9. National Workshop on Fatty Liver and Alcoholic Liver Disease, Chinese Society of Hepatology, Chinese Medical Society, Fatty Liver Expert Committee, Chinese Medical Doctor Association. Guidelines of prevention and treatment for nonalcoholic fatty liver diseases: a 2018 update. J Clin Hepatol. 2018;34(5):947-57.

10. He XX, Su JR, Ma XJ, Lu W, Zhu W, Wang YF, et al. The association between serum growth differentiation factor 15 levels and lower extremity atherosclerotic disease is independent of body mass index in type 2 diabetes. Cardiovasc Diabetol. 2020;19:40.

11. Levey AS, Stevens LA, Schmid CH, Zhang YL, Castro AF 3rd, Feldman HI, et al. A new equation to estimate glomerular filtration rate. Ann Intern Med. 2009;150(9):604-12.

12. Sheng CS, Tian J, Miao Y, Cheng Y, Yang Y, Reaven PD, et al. Prognostic significance of longterm $\mathrm{HbA1} 1 \mathrm{c}$ variability for all-cause mortality in the ACCORD Trial. Diabetes Care. 2020;43(6):1185-90.

13. Touboul PJ, Hennerici MG, Meairs $S$, Adams H, Amarenco P, Desvarieux $\mathrm{M}$, et al. Mannheim intima-media thickness consensus. Cerebrovasc Dis. 2004;18(4):346-9. 
14. Aboyans V, Ricco JB, Bartelink MEL, Björck M, Brodmann M, Cohnert T, et al. 2017 ESC quidelines on the diagnosis and treatment of peripheral arterial diseases, in collaboration with the European Society for Vascular Surgery (ESVS): document covering atherosclerotic disease of extracranial carotid and vertebral, mesenteric, renal, upper and lower extremity arteries Endorsed by: the European Stroke Organization (ESO) The Task Force for the Diagnosis and Treatment of Peripheral Arterial Diseases of the European Society of Cardiology (ESC) and of the European Society for Vascular Surgery (ESVS). Eur Heart J. 2018;39(9):763-816.

15. Cardoso CRL, Leite NC, Moram CBM, Salles GF, et al. Long-term visitto-visit glycemic variability as predictor of micro- and macrovascular complications in patients with type 2 diabetes The Rio de Janeiro Type 2 Diabetes Cohort Study. Cardiovasc Diabetol. 2018;17(1):33.

16. Ma X, Hu X, Zhou J, Hao Y, Luo Y, Lu Z, et al. Glycated albumin is more closely correlated with coronary artery disease than 1,5-anhydroglucitol and glycated hemoglobin A1c. Cardiovasc Diabetol. 2015;14:16.

17. Mihara A, Ohara T, Hata J, Honda T, Chen S, Sakata S, et al. Association between serum glycated albumin and risk of cardiovascular disease in a Japanese community: The Hisayama Study. Atherosclerosis. 2020:311:52-9.

18. Zhao X, Li Q, Tu C, Zeng Y, Ye Y. High glycated albumin is an independent predictor of low response to clopidogrel in ACS patients: a cross-sectional study. Cardiovasc Diabetol. 2020;19(1):171.

19. Jun JE, Lee SE, Lee YB, Ahn JY, Kim G, Jin SM, et al. Glycated albumin and its variability as an indicator of cardiovascular autonomic neuropathy development in type 2 diabetic patients. Cardiovasc Diabetol. 2017;16(1):127.

20. Park SB, Kim SS, Kim IJ, Nam YJ, Ahn KH, Kim JH, et al. Variability in glycated albumin levels predicts the progression of diabetic nephropathy. J Diabetes Complications. 2017;31(6):1041-6.

21. Chen CW, Drechsler C, Suntharalingam P, Karumanchi SA, Wanner C, Berg AH. High glycated albumin and mortality in persons with diabetes mellitus on hemodialysis. Clin Chem. 2017:63(2):477-85.

22. Hashimoto K, Osugi T, Noguchi S, Morimoto Y, Wasada K, Imai S, et al. A1C but not serum glycated albumin is elevated because of iron deficiency in late pregnancy in diabetic women. Diabetes Care. 2010;33(3):509-11.

23. Gorst C, Kwok CS, Aslam S, Buchan I, Kontopantelis E, Myint PK, et al. Long-term glycemic variability and risk of adverse outcomes: a systematic review and meta-analysis. Diabetes Care. 2015;38(12):2354-69.
24. Forbes A, Murrells T, Mulnier $\mathrm{H}$, Sinclair AJ. Mean HbA, HbA variability, and mortality in people with diabetes aged 70 years and older a retrospective cohort study. Lancet Diabetes Endocrinol. 2018;6(6):476-86.

25. Chang CM, Hsieh CJ, Huang JC, Huang IC. Acute and chronic fluctuations in blood glucose levels can increase oxidative stress in type 2 diabetes mellitus. Acta Diabetol. 2012;49(Suppl 1):S171-7.

26. Wei F, Sun X, Zhao Y, Zhang H, Diao Y, Liu Z. Excessive visit-to-visit glycemic variability independently deteriorates the progression of endothelial and renal dysfunction in patients with type 2 diabetes mellitus. BMC Nephrol. 2016;17(1):67.

27. Schisano B, Tripathi G, McGee K, McTernan PG, Ceriello A. Glucose oscillations, more than constant high glucose, induce p53 activation and a metabolic memory in human endothelial cells. Diabetologia. 2011;54:1219-26.

28. Maciejewski ML, Dowd B, Call KT, Feldman R. Comparing mortality and time until death for medicare $\mathrm{HMO}$ and FFS beneficiaries. Health Serv Res. 2001;35:1245-65.

29. Xu Y, Ma X, Shen Y, Wang Y, Zhou J, Bao Y. Increasing waist circumference is associated with decreased levels of glycated albumin. Clin Chim Acta. 2019:495:118-22

30. Wang F, Ma X, Hao Y, Yang R, Ni J, Xiao Y, et al. Serum glycated albumin is inversely influenced by fat mass and visceral adipose tissue in Chinese with normal glucose tolerance. PLoS ONE. 2012;711(11):e51098.

31. Rooke TW, Hirsch AT, Misra S, Sidawy AN, Beckman JA, Findeiss LK, et al. 2011 ACCF/AHA Focused Update of the Guideline for the Management of patients with peripheral artery disease (Updating the 2005 Guideline): a report of the American College of Cardiology Foundation/American Heart Association Task Force on practice guidelines. J Am Coll Cardiol. 2011;58(19):2020-45.

\section{Publisher's Note}

Springer Nature remains neutral with regard to jurisdictional claims in published maps and institutional affiliations.
Ready to submit your research? Choose BMC and benefit from:

- fast, convenient online submission

- thorough peer review by experienced researchers in your field

- rapid publication on acceptance

- support for research data, including large and complex data types

- gold Open Access which fosters wider collaboration and increased citations

- maximum visibility for your research: over $100 \mathrm{M}$ website views per year

At BMC, research is always in progress.

Learn more biomedcentral.com/submissions 